Research Article

\title{
Effect of Basketball Sports on Serum Superoxide Dismutase and Its Relationship with the Nanoparticle Drug Delivery System
}

\author{
Jinzhong Wang ${ }^{1}$ and Zhenhua Guo $\mathbb{D}^{2}$ \\ ${ }^{1}$ Department of Humanities and Social Sciences, Zhejiang Industry Polytechnic College, Shaoxing 312000, Zhejiang, China \\ ${ }^{2}$ Physical Science College of Jishou University, Jishou 416000, Hunan, China \\ Correspondence should be addressed to Zhenhua Guo; guozhenhua@jsu.edu.cn
}

Received 13 August 2020; Revised 17 September 2020; Accepted 21 September 2020; Published 11 November 2020

Academic Editor: Tifeng Jiao

Copyright (c) 2020 Jinzhong Wang and Zhenhua Guo. This is an open access article distributed under the Creative Commons Attribution License, which permits unrestricted use, distribution, and reproduction in any medium, provided the original work is properly cited.

\begin{abstract}
Human survival is impossible without oxygen, and as the body load continues to increase, the need for oxygen intake becomes greater. However, oxygen is also a double-edged sword for the human body. A large number of studies have proved that excessive intake of oxygen might lead to oxygen poisoning. Even under normal oxygen uptake, there is still a certain proportion of SOD conversion in oxygen. Superoxide dismutase (SOD) is one of the main causes of oxygen poisoning and chronic diseases. It is of great significance to study the changes of SOD in a large amount of oxygen environment. However, there are a few research studies in this field at home and abroad. Therefore, this paper puts forward the influence of basketball sports on serum superoxide dismutase (SOD) and its relationship with the nanoparticle drug delivery system. The research of this paper is mainly divided into three parts. The first part is the research of theoretical basis and core concepts. Through this part of the study, this paper shows that exercise can make the human body strong, while controlling the transformation of SOD, and only in this way can we achieve the true meaning of sports health. The second part is the establishment method of the test model of the influence of basketball on SOD and the nanoparticle drug delivery system. In this part, the principle and operation steps of the design method are given in detail. In order to ensure the effect of the experiment, the test standard was established, and the whole process data were recorded for the retrospective study. The third part is the comparative experiment, which includes the influence of different exercise intensities on SOD activity and the preservation stability of nanoparticles. Through the analysis of experimental data, it was found that basketball increased the risk of SOD transformation, but at the same time, using nanoparticles intervention can effectively reduce the harm of SOD to the human body.
\end{abstract}

\section{Introduction}

In the first line of superoxide dismutase, Oberley once said that "life is acquired by taking great risks." This is a wonderful summary of one of the biggest contradictions in the biological world. This huge contradiction is the "duality" of oxygen to the life process, which is caused by the aerobic nature of organisms and the potential harm of oxygen to organisms. As we all know, except anaerobic organisms, oxygen is the basic living condition of all animals, plants, and human beings. Hypoxia will cause harm to the body and even lead to death. However, if the oxygen concentration exceeds the normal level, it will cause harm to the body and endanger life, which is called oxygen poisoning. Looking back on the changes of the earth's surface environment, as the earth's surface atmosphere has changed from reductive to oxidizing, oxygen stress based on oxidative damage to the body has become a major problem of life and death at that time. At that time, most organisms adapted to the reductive environment, and the oxidizing environment became the condition for species extinction. In order to adapt to the oxidative environment, the survival species in the evolution process continue to establish and improve the protection mechanism of antioxidant damage, such as various antioxidant enzyme systems. However, these mechanisms are not perfect. Oxygen stress still 
causes stress on human health. Many chronic traumatic diseases (tumor, cardiovascular disease, degenerative disease, and various inflammations) and aging are related to oxidative damage.

What is the mechanism of oxygen damage in aerobic organisms? In the mid-1950s, American scientists proposed that the damage of oxygen was attributed to the formation of oxygen free radicals. Subsequent experiments showed that oxygen is toxic not only in the high oxygen condition but also in the normal oxygen environment. Under physiological conditions, $1 \%-4 \%$ of them were converted into superoxide radical $\mathrm{O}_{2-}$ and hydrogen peroxide $\mathrm{H}_{2} \mathrm{O}_{2}$. If $\mathrm{O}_{2-}$ and $\mathrm{H}_{2} \mathrm{O}_{2}$ could not be removed in time, the reaction could continue to produce more toxic hydroxyl radical OH. In 1969, experts discovered superoxide dismutase (SOD) and studied its biological significance, which inevitably revealed the free radical mechanism of oxygen effect. The results show that the formation of $\mathrm{O}_{2-}$ is the main factor of oxygen toxicity, and the theory of superoxide radical $\left(\mathrm{O}_{2-}\right)$ of oxygen toxicity is put forward, which greatly promotes the in-depth and systematic research of oxygen metabolism and is a major breakthrough in the history of SOD research. A large number of studies have shown that exercise can increase the body's oxygen uptake. The sharp increase of oxygen uptake not only ensures the exercise ability but also increases the possibility of oxygen poisoning.

Basketball is a popular sport all over the world, which takes into account both competition and entertainment, and is one of the mainstream sports. Under the background that we strongly advocate mass basketball, the research on basketball training and athletes' physical health has become the current research hotspot. However, the current research is too theoretical and does not involve the study of oxygen poisoning, and the research on oxygen poisoning is still blank. In view of this situation, this paper puts forward the research on the influence of basketball sports on serum superoxide dismutase and its relationship with the nanoparticle drug delivery system, hoping to make up for the research blank in this field.

In this part, we mainly focus on the physical characteristics of basketball and the core knowledge of superoxide dismutase and the nanoparticle drug delivery system. Through the research, this paper believes that oxygen uptake is a double-edged sword for the human body. It not only ensures our daily life but also brings us the harm of oxygen poisoning. In the process of treating oxygen poisoning, the nanoparticle drug delivery system plays a key role. Then, the test model of the influence of basketball on SOD and the nanoparticle drug delivery system is established. The model is based on real person samples, and a sports training plan is made by recruiting volunteers. Then, the volunteers were randomly divided with 34 people in each group. The data were recorded throughout the experiment, and the preparation of serum samples and the detection of SOD activity were carried out according to the plan. In the third chapter, the principle and operation steps of the experiment are given in detail. Finally, in order to further verify the actual effect of this model, a number of comparative experiments including the effect of different exercise intensities on SOD activity, the comparison of serum SOD and MDA concentration, and the preservation stability of nanoparticles were carried out. Through the analysis of the experimental data, this paper believes that basketball belongs to a kind of violent exercise, in the process of exercise, oxygen uptake increased, and in the case of constant proportion, the composition of SOD also changed more. The increase of SOD further increases the risk of oxygen poisoning and the occurrence of some chronic diseases. At the same time, the data also showed that, under the intervention of the nanoparticle drug delivery system, SOD could be better inhibited, and after strenuous exercise, the activity and stability of nanoparticles were further improved [1-3].

\section{Theoretical Basis and Core Concepts of This Paper}

\subsection{Characteristics of Basketball}

2.1.1. Project Characteristics. The competitive nature of basketball is produced with the birth of basketball. Scholars put forward the introduction of event group training theory, which fully proves the project characteristics of basketball and plans the antagonistic competition of technical and tactical advantages of basketball in the same competition. Both in the early game with simple rules and in the late game with complete rules, basketball players show the charm of basketball in the game. The results of the game will only promote the development of basketball to a higher level, and basketball is a kind of physical exercise for the purpose of scoring in the shooting competition, which has a strong antagonism to the rapid change of attack and defense rhythm and requires high speed and strength. Thus, basketball is not only simply understood as a skill-based sport but also a sport with high physical requirements for athletes.

2.1.2. Physical Characteristics. The basketball game goes round and round. At a certain time, on the court, the players on both sides try to throw the ball into the other side's basket by attacking. Defense prevents the attacker from throwing the ball into his own basket and getting the ball to continue to attack. According to statistics, in a basketball game, the average distance that the players move on the court is about 3000 meters. The movement of different distances can be simply divided into six basic forms: running with the ball, running backward, walking, jogging, medium-speed running, and fast running. Thus, physical fitness is very important for playing basketball. Therefore, according to the physical fitness of basketball players in the game, moderateintensity and high-intensity sports are the main physical characteristics of basketball $[4,5]$.

\subsection{Physical Quality Characteristics of Basketball Players.} In addition to the common characteristics and competitive sports, the power quality of athletes mainly meets the requirements of basketball quality characteristics. Based on the external quality of speed, the main characteristics of basketball players are fast reaction, fast start, fast stop, fast frequency and speed, action, the overall cohesion of action, 
and the conversion speed of movement. The endurance quality of basketball players mainly includes general endurance quality and special endurance quality, and special endurance quality is the main quality. The agility quality of high-level basketball players is mainly reflected in speed, coordination, and accuracy. The flexibility quality mainly refers to the stretching degree and strong elasticity of each joint of the ligaments. According to the continuous change of the field, the athletes should choose a reasonable position in time, adjust and change the direction and rhythm of the movement, and make a rapid change of technical movements. They skillfully combine various technologies to complete the transformation of offensive and defensive techniques and tactics [6-8].

\subsection{Origin and Classification of SOD. SOD is a key defense} against oxygen toxicity. It is a common and important metal enzyme, which can resist the toxicity of reactive oxygen species. It widely exists in various aerobic and anaerobic organisms, and there is a small amount of SOD in specific anaerobic organisms. About 30 years ago, there was no oxygen in the earth's primitive atmosphere. Primitive life depended on the biosynthesis of sugar absorbed from the environment and glycolysis for energy. There was no oxygen.

SOD was first isolated from bovine red blood cells in 1938. In the process of separating bovine red blood cells step by step, a light blue copper-containing protein was found, which was named blood copper protein. When Melian, Huangchuang, and Huangshengyin studied the decrease of cytochrome $\mathrm{c}$, it was found that copper protein in the blood was originally an enzyme protein. In 1969, he first named it superoxide dismutase (SOD) and further discovered its activity.

On the basis of SOD, it can be divided into three types: (1) copper and zinc, hereinafter referred to as CuZn SOD, blue-green, mainly exists in the cytoplasm and chloroplast matrix of eukaryotic cells, animal blood, liver, milk, plants, leaves, and fruits. It is composed of two subunits, each containing $1 \mathrm{Cu}$ and $\mathrm{Zn}$. There is a synthetic CuZn SOD in the market. (2) Mn, or Mn SOD for short, is purplish red and mainly exists in prokaryotic cells, eukaryotic cells, and the mitochondrial matrix. The Mn SOD in prokaryotic cells is composed of two subunits, each of which contains one manganese. The Mn SOD of eukaryotic cells consists of four subunits. (3) Fe SOD is yellowish brown and mainly exists in prokaryotes and a few plants. It is also composed of two subunits, each containing one Fe [9-11].

\subsection{Physiological Function of SOD}

2.4.1. Improve Resistance to Disease. Because SOD can effectively eliminate $\mathrm{O}_{2-}$ and fight against oxygen poisoning, it plays an important role in prevention of radiation damage and tumor and antiaging and anti-inflammatory processes and has been used abroad to treat various diseases caused by $\mathrm{O}_{2-}$ autoimmune diseases, such as lupus erythematosus, arthritis, and rheumatoid arthritis, senile degenerative diseases, combined with radiotherapy for cancer, and tissue ischemia injury. SOD is very effective in the treatment of autoimmune diseases because it can eliminate $\mathrm{O}_{2-}$, thus effectively inhibiting the speed of lymphocyte chromosome breakage.

2.4.2. Improve Resistance to Inducers. Smoke, radiation, and toxic chemicals can induce the body to produce $\mathrm{O}_{2-}$. SOD can enhance the body's ability to adapt to the external environment and reduce the damage.

\subsubsection{Increase the Adaptability of Large Amount of Exercise.} In the process of excessive exercise such as military training and sports, some tissues and cells in the body will alternately suffer from temporary ischemia and reperfusion injury. In addition, increased lactic acid can lead to muscle fatigue and injury. If you supplement SOD before exercise, it can protect muscle tissue and improve endurance. SOD scavenging free radicals play an important role in the protection of the body, effectively delaying the aging phenomenon caused by free radical damage. It can improve the body's resistance to smoke, radiation, and other free radical-inducing factors, eliminate muscle fatigue, and enhance the adaptability to excessive exercise. Therefore, as a functional food base material, SOD has become a new trend in the application in food and cosmetics $[12,13]$.

\subsection{Characteristics of the Nanoparticle Drug Delivery System.} The nanoparticle drug delivery system is a kind of particle delivery system which can carry out various surface modifications in the diameter of $10-1000 \mathrm{~nm}$. Tumor has better target. Antitumor drugs can improve the stability, drug release, and target delivery and prolong the influence of drugs. In the premise of ensuring the effect, reducing the dosage of drugs and reducing or avoiding the side effects of drugs on normal tissues have been widely studied in the treatment of tumors. The nanoparticle drug delivery system in vivo includes five stages: in vivo circulation (c), accumulation in the tumor site (a), infiltration into tumor (P), absorption into tumor cells (D), and intracellular drug release (R). The ideal therapeutic effect of tumor requires the nanoparticle drug delivery system to complete capir in vivo.

\subsection{Advantages of the Nanoparticle Drug Delivery System.} The nanoparticle drug delivery system has significant advantages.

(1) Sustained release of drug and controlled release: drug release can be controlled due to the different types, properties (such as molecular weight and ratio), and preparation process of carrier materials.

(2) It can improve the absorption and bioavailability of drugs. Because of the highly dispersed nanoparticles, the surface area is larger because of their special surface properties, such as biological adhesives and electrical affinity. It can increase the contact time and the contact area of the drug absorption area. Combined with drug nanoparticles, it has an obvious 
protective effect, so it can improve the absorption and bioavailability of drugs.

(3) Targeted transport: the size of nanoparticles is related to their targeting ability, with which they can target the tissues rich in phagocytes such as the liver, spleen, lung, and lymph. Particles less than $5 \mu \cdot \mathrm{M}$ can be captured by the pulmonary capillary bed and easily engulfed by the reticuloendothelial system (RES). Nanoparticles smaller than $300 \mathrm{~nm}$ can be used for blood circulation, and nanoparticles smaller than $100 \mathrm{~nm}$ can be targeted at tissues or organs, such as the spleen or bone marrow.

(4) On the premise of ensuring the efficacy, the dosage can be reduced to avoid or reduce the side effects of the drug.

(5) Some new routes of administration can be established, such as local administration in vivo, mucosal absorption administration, and oral administration.

A large number of experiments also show that the nanoparticle drug delivery system can improve the stability of drugs (especially water-soluble drugs and genetic drugs) and avoid drug degradation leakage. At the same time, the system can improve the targeting of drugs to the tumor site, so as to improve the curative effect and reduce the side effects. Based on the abovementioned advantages, the nanoparticle drug delivery system has been widely considered. Nanodrugs can selectively guide the liver lesions, merge or be phagocytized by the hepatocytes, reduce the distribution of drugs in the body, and improve the treatment index, which has become an entry point of innovative drug research for hepatitis B. At present, the research of nuclear drugs and interferon nano-liver-targeting agents is actively carried out at home and abroad. However, these targeted drugs are often limited by their own toxicity and selectivity, and the proportion of ideal therapeutic effects is still very low. After drug treatment, immune tolerance is broken, and various adverse reactions and drug resistance are almost inevitable $[14,15]$.

\section{Test Model of the Influence of Basketball on SOD and the Nanoparticle Drug Delivery System}

3.1. Experimental Objects and Methods. Basketball players of a normal university were randomly divided into an experimental group and control group, a total of 68 people, 34 in each group.

3.2. Training Method. Before the start of a Provincial College Basketball League, 68 players trained for four weeks. The training is divided into three stages: the first stage focuses on improving basic skills and physical quality, the second stage is the improvement of the technical and tactical level, and the third stage is to solve the exposed problems through the actual combat test training results. The training content is the combination of physical training and basketball skills and tactics, including strength, speed, endurance, agility, and coordination, three times a week, about 4 hours a day, and basic training such as dribbling and shooting in the morning. In the afternoon, the training includes 35 minutes of preparation and 85 minutes of formal training. After the formal training, physical training begins, organizing activities for 15 minutes, mainly to relax and stretch the ligament, and the two groups of training content are the same.

3.3. Experimental Equipment. This experiment mainly uses 20 kinds of main experimental equipment including the PCR gene amplification instrument, as shown in Table 1.

3.4. Experimental Reagents. Nine kinds of main reagents including a Promega DNA kit were used in this experiment, as shown in Table 2.

3.5. Preparation of Serum Samples. After fasting for 8 hours before and after treatment, fasting venous blood routine and biochemical indexes were measured in the morning. The other $5 \mathrm{~m} 1$, which was not anticoagulant therapy, was set at $36^{\circ} \mathrm{C}$ thermostat for more than 35 minutes and, then, centrifuged at a speed of $3500 \mathrm{rpm}$. After centrifugation for 15 minutes, the serum was separated from EP and sealed with tube packing. The samples were stored at $-68^{\circ} \mathrm{C}$ after freezing in a low-temperature refrigerator.

\subsection{Determination of SOD Activity}

3.6.1. Preparation of $80 \mathrm{mmol} / \mathrm{L}$ Phosphate Buffer ( $p H$ 7.6). $16.28 \mathrm{~g} \mathrm{~K}_{2} \mathrm{HPO}_{4} 3 \mathrm{H}_{2} \mathrm{O}$ and $0.95 \mathrm{~g} \mathrm{KH}_{2} \mathrm{PO}_{4}$ were weighed and adjusted to $1.5 \mathrm{~L}$ with acid or alkali, and the $\mathrm{pH}$ was adjusted to 7.6 .

3.6.2. $0.2 \mathrm{~mol} / \mathrm{l}$ Photoamine Hydrochloride Solution (Currently Used). According to the quantity requirements of the sample detection, the appropriate amount of photoamine hydrochloride was dissolved in the appropriate amount of distilled water to prepare $0.00581 \mathrm{~g} / 1$ photoamine hydrochloride solution.

For the determination of more than 20 samples, the specific preparation method is as follows: before determining the sample, the amount of photoamine hydrochloride solution used is estimated. For example, the amount of solution needed to determine 20 samples is $0.06^{*}$ $35=1.8 \mathrm{ml}$. Due to some errors in the experiment, $1.79 *$ $0.00581=0.0258 \mathrm{~g}$ was accurately weighed, that is, $0.0232 \mathrm{~g}$ hydrochloric acid was added into $1.81 \mathrm{ml}$ distilled water through amine.

3.6.3. $80 \mathrm{mmol} / \mathrm{L}$ Yellow Silver Solution (Present and Present). According to the requirements of sample detection amount, appropriate amount of the baicalin powder was dissolved in appropriate amount of distilled water to prepare $0.0284 \mathrm{~g} / \mathrm{l}$ xanthine solution. 
TABLE 1: List of the main experimental equipment.

\begin{tabular}{lc}
\hline Instrument name & Manufacturer \\
\hline Centrifuge tgl-16g & Shanghai Anting Scientific Instrument Factory \\
PCR gene amplification instrument & Tianlong Technology Co., Ltd. \\
Power supply for dyy-6c electrophoresis instrument & Beijing Liuyi Instrument Factory \\
Wd-9403b ultraviolet instrument & Beijing Liuyi Instrument Factory \\
Model 752 ultraviolet spectrophotometer & Tianpu Analytical Instrument \\
Digital camera & Mingji Electric Communication Co., Ltd. \\
Constant temperature water bath hh-4 & Electronic Instrument Co., Ltd. \\
Electronic balance & Yueping Scientific Instrument Co., Ltd. \\
Co mix mixer & Shanghai Lainte Electronics \\
$-80^{\circ}$ C low-temperature refrigerator & Beijing Tiandi Jingyi Company \\
Pipette gun & Bioengineering \\
Suction head & Bioengineering \\
Centrifugal tube & Bioengineering \\
PCR tube & Bioengineering \\
Abi3100 DNA sequencer & Bioengineering \\
Body composition analyzer & Imported from South Korea \\
Automatic blood cell analyzer & Jinan Glite Technology \\
Cardiopulmonary function tester & Made in Germany \\
Treadmill for the exercise load test & Contec \\
Rs 800 heart rate meter & Made in Finland
\end{tabular}

TABLE 2: List of main reagents.

\begin{tabular}{lc}
\hline Reagent name & Manufacturer \\
\hline Promega DNA & Plomeg \\
Anhydrous ethanol & Bioengineering \\
Anhydrous isopropanol & Bioengineering \\
PCR primer synthesis & Bioengineering \\
Taq polymerase sc0010 & Bioengineering \\
dNTP mixture solution & Bioengineering \\
Regular Agarose G-10 & Biowest Agarose \\
DNA marker GM343 & Bioengineering \\
BsaWI R0567S & NeWin Biotechnology \\
\hline
\end{tabular}

A specific method for the determination of more than 20 samples was established with hydroxylamine solution as the solvent.

3.6.4. Preparation of $0.165 \mathrm{~mol} / \mathrm{L} \mathrm{NaOH}$ Solution. $0.9 \mathrm{~g}$ $\mathrm{NaOH}$ was dissolved in $50 \mathrm{ml}$ water and diluted 5 times to $0.145 \mathrm{~mol} / \mathrm{l} \mathrm{NaOH}$.

\subsection{2 u/l Xanthine Oxidase Solution (to Be Prepared} Fresh and Stored at $5^{\circ} \mathrm{C}$ ). More than 20 samples of xanthine oxidase were determined.

The amount of enzyme used is estimated before determining the sample. For example, the enzyme amount needed for determination of 20 samples is $0.06^{*} 20=1.9 \mathrm{ml}$.

In the experiment, the stored enzyme solution was divided into $250 \mathrm{UL} /$ tube and diluted 85 times before use. When the calculated enzyme amount was $1.5 \mathrm{ml}$, about $25 \mathrm{ul}$ $\left(0.025^{*} 45=1.68 \mathrm{ml}\right)$ of enzyme solution was absorbed, and the buffer solution was diluted 85 times.

\subsection{Determination of CAT Activity}

3.7.1. Reagent Preparation. Reaction substrate solution: $35 \%$ $\mathrm{H}_{2} \mathrm{O}_{2} 0.62 \mathrm{ml}$ is taken, distilled water is added to $55 \mathrm{ml}, 5 \mathrm{ml}$ is taken, and $0.06 \mathrm{~mol} / \mathrm{l}$ phosphate buffer solution is added with $\mathrm{pH}=7.5,25 \mathrm{ml}$. The $\mathrm{OD}$ value of $2 \mathrm{~cm}$ was measured at $280 \mathrm{~nm}$, and the ratio of $\mathrm{H}_{2} \mathrm{O}_{2}$ diluent to phosphate buffer solution was adjusted to make the OD value between 0.8 and 0.95 .

3.7.2. Activity Determination. High-efficiency cyanohydrin (260490980, 2000, and $3950 \mathrm{~g} / \mathrm{L})$, avermectin $(50,90180$, and $350680 \mathrm{~g} / \mathrm{L})$, and phoxim $(680,1500,2800,5200$, and $12000 \mathrm{~g} / \mathrm{L}$ ) were incubated with $0.2 \mathrm{ml}$ of protoenzyme solution at room temperature for $10 \mathrm{~min}$. Then, the hydrogen peroxide substrate solution preheated to $30^{\circ} \mathrm{C}$ is immediately added into $5 \mathrm{ml}$, and the OD value is determined at $280 \mathrm{~nm}$. The test is conducted once in every 35 seconds 6 consecutive times.

3.8. Preparation of Albumin Nanoparticles. $\mathrm{As}_{2} \mathrm{O}_{3}$ and a small amount of $1 \mathrm{~mol} / \mathrm{L} \mathrm{NaOH}$ solution were heated and dissolved, and serum albumin was dissolved after cooling. After the solution was completely dissolved, the $\mathrm{pH}$ value was slightly alkaline. Anhydrous ethanol was added slowly at room temperature and stirred for half an hour, and then, glutaraldehyde solution was added slowly until turbid. The reaction liquid was poured into the centrifuge tube, centrifuged in a centrifuge $(8500 \mathrm{rpm})$, washed three times, and dried in vacuum. Calcitonin was dissolved in the aqueous solution of serum albumin, and the albumin nanoparticles loaded with calcitonin were prepared according to the abovementioned operation.

3.9. Nanoprecipitation Method. Part of $60 \mathrm{mg} 15 \%$ PEGPLGA (60/60) was accurately weighed, and $10 \mathrm{ml}$ organic solvent acetone was added to form an organic phase. After ultrasonic treatment for 10 minutes, PEG-PLGA was uniformly dissolved in acetone and, then, (drug) was dissolved in $15 \mathrm{ml}$ acetone to fully dissolve the organic phase. $0.3 \mathrm{~g}$ 
CTAB was dissolved in $60 \mathrm{ml}$ water, and $0.30 \mathrm{~g}$ PVA (P200 or Twan 100) was added into CTAB aqueous solution under heating conditions. After the probe was completely dissolved, ultrasonic treatment was conducted for 5 minutes to form an aqueous phase (W). Under the condition of a magnetic stirrer (1300 rpm, $35 \mathrm{~min}$ ), the organic phase was added into the aqueous phase, and the stirring was accelerated by using a cell crushing device. After $35 \mathrm{~min}$ of magnetic stirring, the organic solvent acetone was removed by using a rotating evaporator to obtain the solution of nanoparticles. The size and zeta potential of nanoparticles were measured.

3.10. Determination of Particle Size Potential. Based on the principle of laser diffraction, Wally Wood measured the average particle size and particle size distribution of nanoparticles by the light scattering method and measured zeta potential to determine whether the prepared nanoparticles were cationic nanoparticles.

3.11. Statistical Processing of Data. SPSS16.0 statistical software was used to process and analyze all the data. The Hardy-Weinberg $(\mathrm{H}-\mathrm{W})$ formula was used to determine the distribution frequency of genotype and allele. The $X^{2}$ test was used to compare the count between the two groups. The independent sample $t$-test was used to compare the exercise ability index among different genotypes. The significant level was $p<0.05$, and the extremely significant level was $p<0.01$.

\section{Test Results and Analysis}

4.1. Effect of Different Exercise Intensities on SOD Activity. According to the analysis results in Figure 1, compared with the control group, the serum SOD activity of the experimental group under different training loads was significantly increased $(p<0.1)$, and compared with the control group before training, the basketball training plan with different loads significantly increased the SOD activity of the experimental group $(p<0.05)$. The activity of SOD in the medium-intensity training group was significantly higher than that in the lowintensity training group and high-intensity test stage $(p<0.05)$. Compared with high intensity and low intensity, SOD also increased, and the difference was statistically significant $(p<0.05)$. Through the test of exhausted exercise of two groups of athletes, the analysis of data shows that the activity of SOD changes with different exercise intensities. Specifically, SOD activity increases with the increase of exercise intensity, but its activity also has a peak value. In moderate exercise intensity, the body load is the largest and oxygen uptake is the largest, so the SOD activity is higher.

4.2. Comparison of Serum SOD Concentration. According to the experimental results in Figure 2, the concentration of serum SOD in the experimental group was significantly higher than that in the control group. After 24 hours of exercise, the concentration of MDA was significantly lower than that of the control group after 24 hours of exercise, and the concentration of T-AOC was significantly higher than that of the control group before and after exercise. There was no significant difference in serum SOD, MDA, and T-AOC between the two groups. It can be seen that basketball has a great influence on SOD, especially in the state after sports. Compared with the control group, the experimental group showed stronger cardiac function, which was mainly due to the increase of oxygen uptake, which enhanced the exercise ability of the body. But, at the same time, according to the basic theory, excessive oxygen intake, in which the proportion of SOD will also increase significantly, increases the risk of oxygen poisoning.

4.3. PLA-PLL-RGD Concentration. The experimental results in Figure 3 show that the particle size increases with the increase of PLA-PLL-RGD concentration, but the entrapment efficiency increases from low to high. During emulsification, PLA-PLL-RGD dissolves in organic solvent to form an organic phase, which diffuses into a water phase of the dispersion medium. When the concentration of PLAPLL-RGD increases, the amount of PLA-PLL-RGD diffused increases. Under the same other conditions, high concentration of PLA-PLL-RGD is not easy to disperse, and it easy to produce agglomerated and large-sized nanoparticles. At the same time, with the increase of PLA-PLL-RGD concentration, the viscosity of the organic phase increased and the diffusion speed slowed down. The higher concentration and slower diffusion speed make PLA-PLL-RGD agglomerate easily to form larger micelle particles, which makes the size of the prepared nanoparticles to increase, and it is difficult to form uniform small particles. The results of this experiment, combined with the correlation of SOD, show that although exercise increases the activity of SOD, due to the intervention of nanoparticles, it can effectively reduce the harm of SOD to the human body.

4.4. Preservation Stability of Nanoparticles. The discovery of nanoparticles is, indeed, gratifying, but whether the system can be stably preserved deserves further exploration. In this experiment, the activity, particle size, and zeta potential of SOD were measured regularly to explore the stability of preservation.

It can be seen from the analysis results in Figure 4 that the SOD activity detected in the experimental group remained at a high level after 30 days, with no loss. Although the enzyme activity fluctuated slightly in the previous week, the enzyme activity increased significantly in the first three days, which may be due to the temporary recovery of SOD saturation after the reaction. Compared with the control group, the stability of SOD activity was significantly improved after basketball training. Combined with Figures 3 and 2, the particle size and potential of SOD had, almost, no change during the detection time, indicating that SOD in the experimental group could be stored in the refrigerator at $4^{\circ} \mathrm{C}$ for, at least, one month. The experimental results also remind us that strong exercise may lead to the generation of chronic diseases because after exercise, the stability of SOD enzyme in the body will be improved, which can play a role 


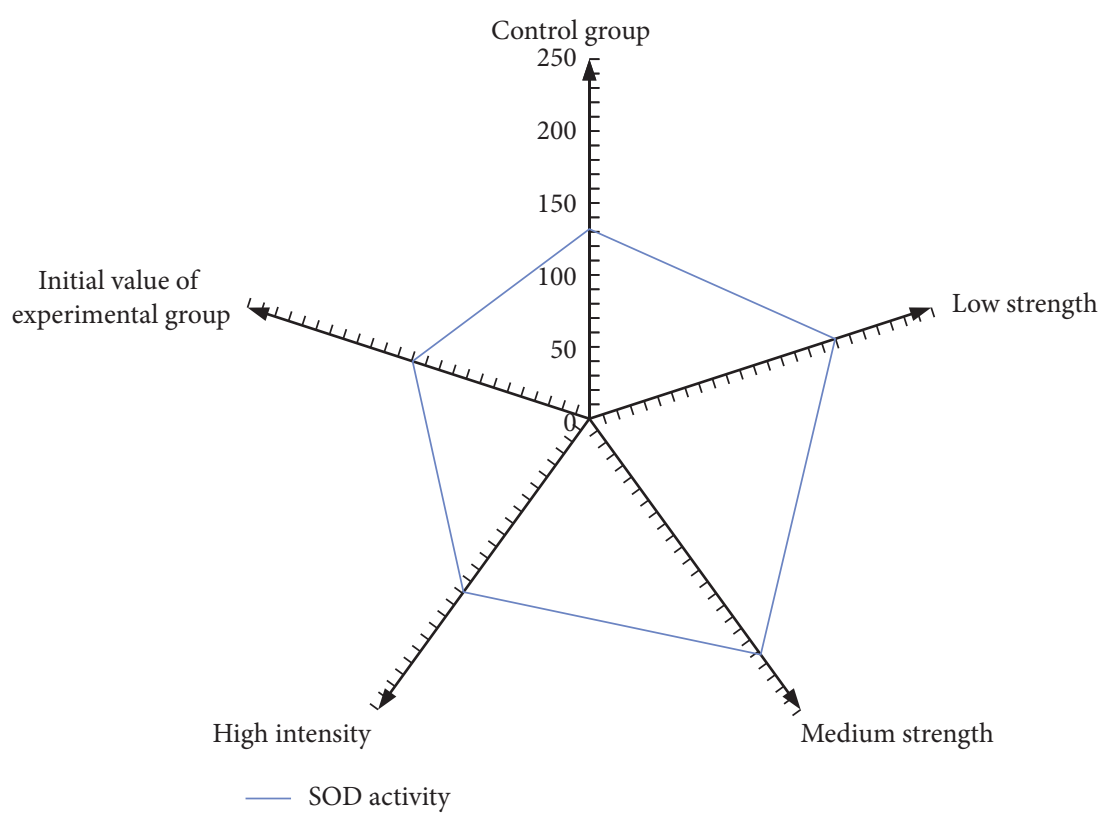

FIGURE 1: Statistical analysis of the effect of different exercise intensities on SOD activity in each group.

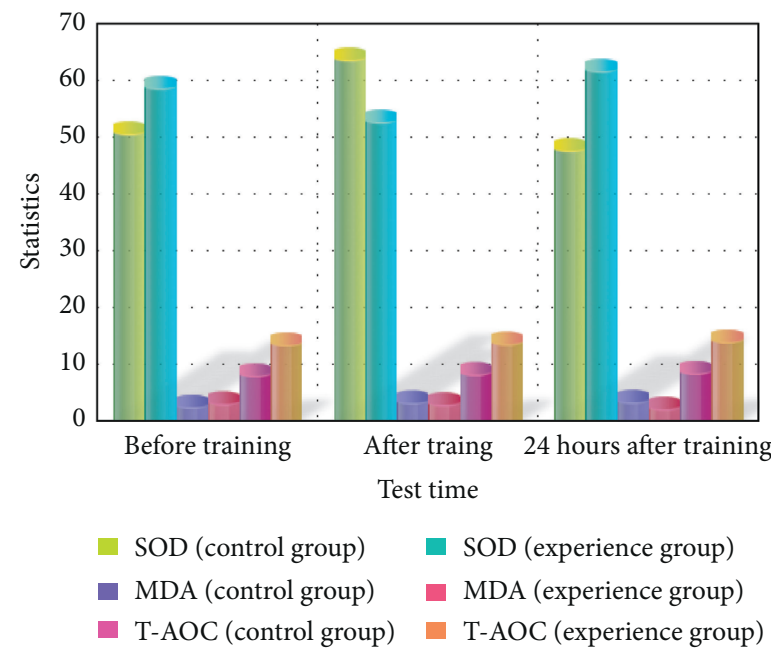

FIGURE 2: Statistical analysis of serum SOD and MDA concentrations in each group after the test.

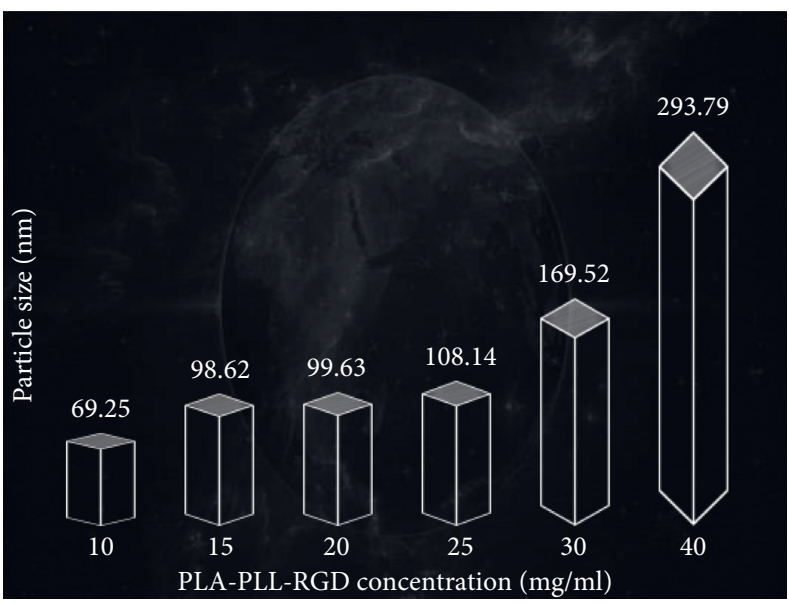

FIGURE 3: Statistical analysis of the effect of pla-pll-rgd concentration on SOD activity. 


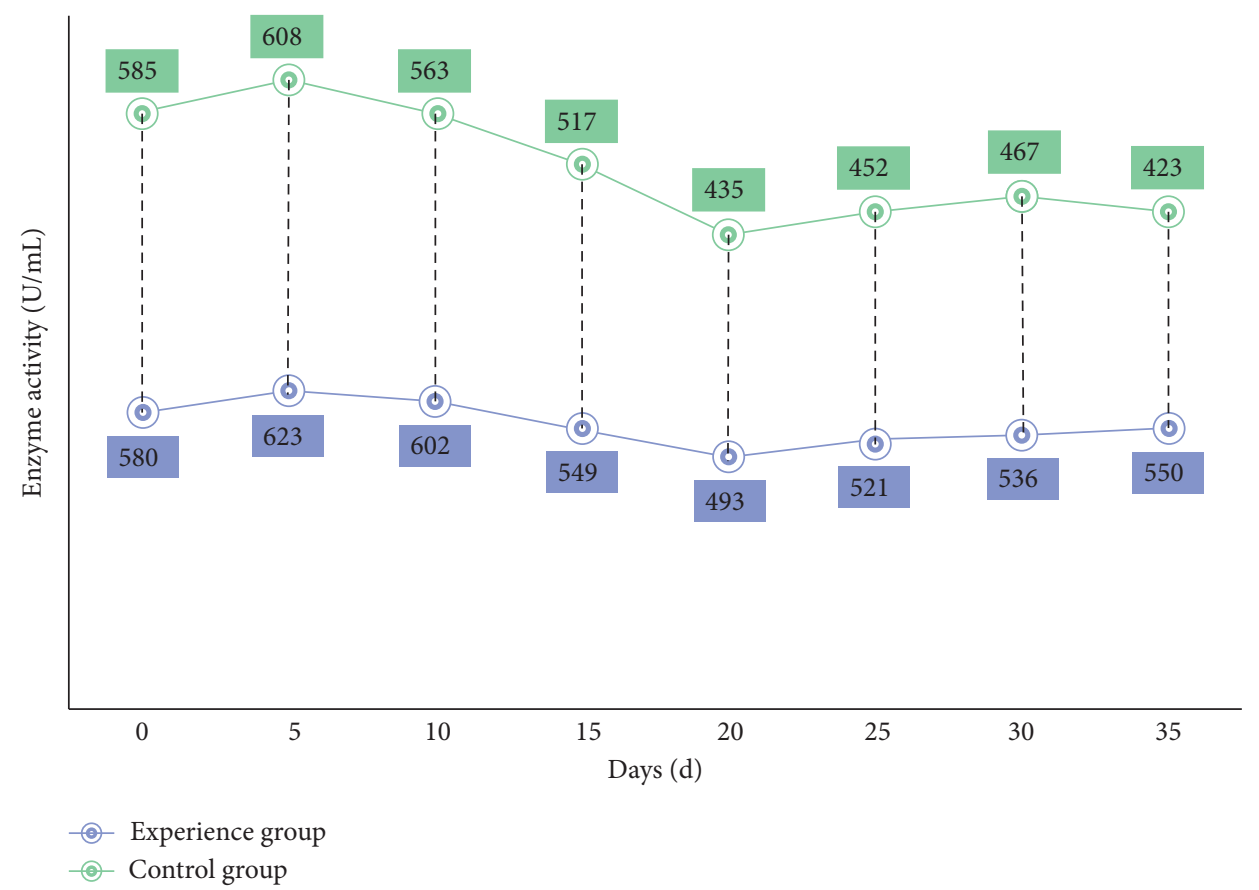

FIGURE 4: Statistical analysis of the preservation stability of nanoparticles in each group of experimental samples.

for a longer time, and the damage to the human body becomes longer, more likely to lead to various diseases and hidden dangers.

\section{Conclusions}

In the past, the research reports on oxygen uptake and oxygen poisoning were mostly focused on extreme exercise under severe state. Extreme sports need a lot of oxygen to support, and the existing theoretical knowledge has shown that the more the oxygen intake, the higher the activity of SOD. Although exercise can improve the metabolism of the body and enhance their own ability to exercise, when the SOD activity is too high, it is accompanied by the possibility of oxygen poisoning. But, in our daily life, many sports in our lives also have such dangers, such as the popular basketball game. At present, there is almost no research on basketball and SOD activity in China, but the research on the effect of basketball on serum SOD and its relationship with the nanoparticle drug delivery system proposed in this paper has made up for the blank in this field to a certain extent. The core aim of this study is to detect the activity of SOD in the state of basketball training and to intervene the experimental body with nanoparticles, so as to reduce the harm of SOD to human body through the drug delivery system. In the third chapter of this paper, the principle of experimental design and specific operation steps are given in detail. Through the research, this paper preliminarily believes that the intensity of basketball is closely related to the activity of SOD. In order to verify the conjecture of this paper, a comparative experiment is carried out in the last part of this paper. Through the analysis of the experimental data, it can be seen that, with the increase in exercise intensity, the SOD activity of the experimental group also gradually increased and, especially, reached the peak value under the condition of moderate exercise intensity. With the increase in SOD activity, the stability of SOD was also greatly improved and it could be stored for more than 30 days at $4^{\circ} \mathrm{C}$. This research has achieved ideal results and made a contribution to the research in this field.

\section{Data Availability}

The data used in this article can be obtained from the corresponding author upon request.

\section{Conflicts of Interest}

The authors declare no conflicts of interest.

\section{Authors' Contributions}

All authors read and approved the manuscript.

\section{Acknowledgments}

This work was supported by Zhejiang Education Department Project (Y201122419).

\section{References}

[1] J. Padulo, G. Laffaye, M. Haddad et al., "Repeated sprint ability in young basketball players: one vs. two changes of direction (part 1)," Journal of Sports Sciences, vol. 33, no. 14, pp. 1480-1492, 2015.

[2] A. Bekiari, S. Perkos, and V. Gerodimos, "Verbal aggression in basketball: perceived coach use and athlete intrinsic and extrinsic motivation," Journal of Physical Education and Sport, vol. 15, no. 1, pp. 96-102, 2015. 
[3] B. T. Mccormick, J. C. Hannon, M. Newton, B. Shultz, N. Miller, and W. Young, "Comparison of physical activity in small-sided basketball games versus full-sided games," International Journal of Sports Ence \& Coaching, vol. 7, no. 4, pp. 689-697, 2016.

[4] W. Chen, T. Lao, J. Xia et al., "Gameflow: narrative visualization of NBA basketball games," IEEE Transactions on Multimedia, vol. 18, no. 11, pp. 2247-2256, 2016.

[5] R. C. Bulson, K. J. Ciuffreda, J. Hayes, and D. P. Ludlam, "Effect of retinal defocus on basketball free throw shooting performance," Clinical and Experimental Optometry, vol. 98, no. 4, pp. 330-334, 2015.

[6] A. Benjaminse, B. Otten, A. Gokeler, R. L. Diercks, and K. A. P. M. Lemmink, "Motor learning strategies in basketball players and its implications for ACL injury prevention: a randomized controlled trial," Knee Surgery Sports Traumatology Arthroscopy Official Journal of the Esska, vol. 25, no. 8, pp. 1-12, 2015.

[7] R. Rienhoff, L. Fischer, B. Strauss, J. Baker, and J. Schorer, "Focus of attention influences quiet-eye behavior: an exploratory investigation of different skill levels in female basketball players," Sport, Exercise, and Performance Psychology, vol. 4, no. 1, pp. 62-74, 2015.

[8] W. J. Markwick, S. P. Bird, J. J. Tufano, L. B. Seitz, and G. G. Haff, "The intraday reliability of the reactive strength index calculated from a drop jump in professional men's basketball," International Journal of Sports Physiology and Performance, vol. 10, no. 4, pp. 482-488, 2015.

[9] B. Sookkheo, S. Sinchaikul, H. Thannan, O. Thongprasong, and S. T. Chen, "Proteomic analysis of a thermostable superoxide dismutase from bacillus stearothermophilus TLS33," Proteomics, vol. 2, no. 9, pp. 1311-1315, 2015.

[10] B. M. Morrison, W. G. Janssen, J. W. Gordon, and J. H. Morrison, "Time course of neuropathology in the spinal cord of g86r superoxide dismutase transgenic mice," Journal of Comparative Neurology, vol. 391, no. 1, pp. 64-77, 2015.

[11] M. A. Khan, C. S. Thompson, J. Y. Jeremy, F. H. Mumtaz, and R. J. Morgan, "The effect of superoxide dismutase on nitric oxide-mediated and electrical field-stimulated diabetic rabbit cavernosal smooth muscle relaxation," BJU International, vol. 87, no. 1, pp. 98-103, 2001.

[12] S. L. Archer, G. Marsboom, G. H. Kim et al., "Epigenetic attenuation of mitochondrial superoxide dismutase 2 in pulmonary arterial hypertension: a basis for excessive cell proliferation and a new therapeutic target," Circulation, vol. 121, no. 24, pp. 2661-2671, 2010.

[13] K. Fujita, M. Yamauchi, K. Shibayama, M. Ando, M. Honda, and Y. Nagata, "Decreased cytochrome c oxidase activity but unchanged superoxide dismutase and glutathione peroxidase activities in the spinal cords of patients with amyotrophic lateral sclerosis," Journal of Neuroence Research, vol. 45, no. 3, pp. 276-281, 2015.

[14] Q. Gu, W. Chen, F. Duan, and R. Ju, "Fabrication of a nanodrug delivery system based on layered rare-earth hydroxides integrating drug-loading and fluorescence properties," Dalton Transactions, vol. 45, no. 30, pp. 12137-12143, 2016.

[15] Y. Dai and F. Xin, "Progress in studies on embryo toxicity of nano drug delivery system," Journal of Adverse Drug Reactions, vol. 17, no. 1, pp. 44-48, 2015. 